\section{Obstructive sleep apnoea and cardiovascular calcification}

\author{
Magnus Bäck, ${ }^{1,2}$ Françoise Stanke-Labesque ${ }^{3,4,5}$
}

The increased cardiovascular risk associated with obstructive sleep apnoea (OSA) has been well established, ${ }^{1}$ but the mechanisms involved remain to be elucidated. The study by Lutsey et $a l^{2}$ published in this issue of Thorax adds another piece to the puzzle of the link between OSA and cardiovascular risk by showing significant associations between parameters of sleep-disordered breathing and coronary atherosclerotic calcification.

The initiation and progression of atherosclerosis is characterised by an accumulation of lipids and lipoproteins within the vascular wall, leading to immune activation and the recruitment of inflammatory cells. ${ }^{3}$ The resulting atherosclerotic plaques may remain silent for a long time before plaque destabilisation occurs, leading to plaque rupture, vessel occlusion and, if in a coronary artery, myocardial infarction. Prognostic markers are of particular interest to identify patients at risk of plaque rupture and who would benefit from preventive actions and medical treatments. In this context, OSA may represent an important modifiable cardiovascular risk factor.

In addition to lipid accumulation and inflammation, vascular calcification plays an important role in atherosclerosis. Although initially considered purely degenerative, atherosclerotic calcification is in fact an active process, which involves calcium deposits, procalcifying particles and a phenotypic transdifferentiation of vascular smooth muscle cells towards an osteoblastic phenotype. Recently, special attention has been paid to the pattern of punctate vascular calcification, since such microcalcifications may be a site of plaque destabilisation and drive plaque rupture.

Scoring the amount of calcium in the coronary arteries by means of CT provides a non-invasive measure of the total atherosclerosis burden. Increased coronary

\footnotetext{
${ }^{1}$ Translational Cardiology, Karolinska Institutet, Stockholm, Sweden; ${ }^{2}$ Department of Cardiology, Karolinska University Hospital, Stockholm, Sweden; ${ }^{3}$ Université Grenoble Alpes, Grenoble, France; ${ }^{4}$ INSERM U1042, HP2, Grenoble, France; ${ }^{5}$ Department of Pharmacology, CHU, Grenoble, France

Correspondence to Dr Magnus Bäck, Center for Molecular Medicine, L8:03, Karolinska University Hospital, 17176 Stockholm, Sweden;

Magnus.Back@ki.se
}

artery calcification (CAC) has been associated with increased cardiovascular risk and shown to provide further prognostic information in addition to traditional cardiovascular risk factors. Evidence is now accumulating linking sleep disturbances with increased CAC, ${ }^{2} 45$ and inflammation may represent an important factor when it comes to OSA-induced atherosclerotic calcification.

The association of increased sleep fragmentation and low sleep quality with increased $\mathrm{CAC}^{2} 5$ implicates sympathetic nervous activation, which in turn may activate inflammatory circuits. However, it is, above all, the typical breathing pattern in OSA causing an intermittent hypoxia, which is a potent proinflammatory stimulus. ${ }^{1}$ Among the inflammatory mediators explored as putative links between OSA and increased cardiovascular risk can be mentioned, for example, lipid mediators of inflammation, such as leukotrienes. ${ }^{6}$ Subjects with OSA exhibit an increased leukotriene production, ${ }^{7}$ and signalling through leukotriene receptors increases experimental atherosclerosis. ${ }^{8}$ Moreover, leukotriene signalling has also been associated with cardiovascular calcification, ${ }^{9}$ which in the context of the results of Lutsey et $a l^{2}$ opens up for further exploration of leukotrienes and other inflammatory mediators in OSA-induced atherosclerotic calcification. Interestingly, the use of the leukotriene receptor antagonist montelukast in asthma has, in addition, been associated with a decreased cardiovascular risk, ${ }^{10}$ further reinforcing the therapeutic implications of this pathway. This point is of particular clinical relevance since 6 months of treatment with CPAP, which is the reference treatment of OSA, does not reduce the plasma levels of either CRP or several proinflammatory mediators in patients with severe OSA. ${ }^{11} 12$

Although significant associations between OSA and CAC are reported in this issue of Thorax, ${ }^{2}$ it should be taken into consideration that only severe OSA remained significantly correlated with CAC after adjustment for traditional cardiovascular risk factors. Likewise, a previous study found significant associations with CAC only in non-obese OSA subjects. ${ }^{4}$ This illustrates the complexity of the interaction between sleep-disordered breathing and atherosclerotic calcification, and the multifactorial participation of the increased cardiovascular risk associated with OSA.

Taken together, although the mechanisms linking OSA to cardiovascular calcification remain to be established, the observed association of OSA with $\mathrm{CAC}^{2}$ reinforces the proatherogenic effects of sleep-disordered breathing and intermittent hypoxia. Nevertheless, multiple comorbidities of patients with OSA must be taken into consideration when studying OSA-associated cardiovascular risk and calcification. The challenges for the future perspectives in this domain lie in the identification of patients with OSA at particular risk of plaque rupture and in the development of specific preventive strategies targeting the pathways of cardiovascular calcification induced by OSA and intermittent hypoxia.

\section{Competing interests None.}

Provenance and peer review Commissioned; internally peer reviewed.

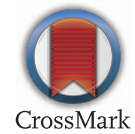

To cite Bäck M, Stanke-Labesque F. Thorax 2015;70:815-816.

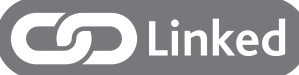

http://dx.doi.org/10.1136/thoraxjnl-2015-206871

Thorax 2015;70:815-816.

doi:10.1136/thoraxjnl-2015-207476

\section{REFERENCES}

1 Levy P, Tamisier R, Arnaud C, et al. Sleep deprivation, sleep apnea and cardiovascular diseases. Front Biosci (Elite Ed) 2012;4: 2007-21.

2 Lutsey PL, McClelland RL, Duprez D, et al. Objectively measured sleep characteristics and prevalence of coronary artery calcification: the Multi-Ethnic Study of Atherosclerosis Sleep study. Thorax 2015;70:880-7.

3 Bäck M, Hansson GK. Anti-inflammatory therapies for atherosclerosis. Nat Rev Cardiol 2015; 12:199-211.

4 Luyster FS, Kip KE, Aiyer AN, et al. Relation of obstructive sleep apnea to coronary artery calcium in non-obese versus obese men and women aged 45-75 years. Am J Cardiol 2014;114: 1690-4.

5 King CR, Knutson KL, Rathouz PJ, et al. Short sleep duration and incident coronary artery calcification. JAMA 2008;300:2859-66.

6 Stanke-Labesque F, Pepin JL, Gautier-Veyret E, et al. Leukotrienes as a molecular link between obstructive sleep apnoea and atherosclerosis. Cardiovas Res 2014;101:187-93.

7 Stanke-Labesque F, Bäck M, Lefebvre B, et al. Increased urinary leukotriene $\mathrm{E} 4$ excretion in obstructive 


\section{Editorial}

sleep apnea: effects of obesity and hypoxia. J Allerg Clin Immunol 2009;124:364-70, 70 e1-2.

8 Ketelhuth DF, Hermansson A, Hlawaty $\mathrm{H}$, et al. The leukotriene B4 receptor (BLT) antagonist BIIL284 decreases atherosclerosis in ApoE-1- mice.

Prostaglandins Other Lipid Mediat. Published Online

First: 4 Jun 2015. doi:10.1016/j.

prostaglandins.2015.05.007
9 Nagy E, Andersson DC, Caidahl K, et al. Upregulation of the 5 -lipoxygenase pathway in human aortic valves correlates with severity of stenosis and leads to leukotriene-induced effects on valvular myofibroblasts. Circulation 2011;123: 1316-25.

10 Ingelsson E, Yin L, Bäck M. Nationwide cohort study of the leukotriene receptor antagonist montelukast and incident or recurrent cardiovascular disease. J Allergy Clin Immunol 2012;129: 702-07 e2.

11 Chirinos JA, Gurubhagavatula I, Teff K, et al. CPAP, weight loss, or both for obstructive sleep apnea. N Engl J Med 2014;370:2265-75.

12 Stradling JR, Craig SE, Kohler M, et al. Markers of inflammation: data from the MOSAIC randomised trial of CPAP for minimally symptomatic OSA. Thorax 2015;70:181-2. 